\title{
Partial Regularity for the Solutions to Nonlinear Parabolic Systems (*).
}

\author{
M. GTAQUTNTA (**) - E. GTUSTT (***)
}

Sunto. - Si estendono a sistemi non lineari di tipo parabolico alcuni risultati di regolarità parziale delle soluzioni di sistemi ellittici.

\section{1. - Introduction.}

The aim of this paper is to extend the results and the methods of [6] to nonlinear parabolic systems of partial differential equations:

$$
\sum_{\alpha=1}^{N} \int_{A} u^{\alpha} \varphi_{i}^{\alpha} d z=\sum_{\alpha, \beta=1}^{N} \sum_{i, j=1}^{n} \int_{A} a_{i j}^{\alpha \beta}(z, u) u_{x_{j}}^{\beta} \varphi_{x_{i}}^{\alpha} d z, \quad \forall \varphi \in C_{0}^{\infty}\left(A: \mathbb{R}^{N}\right)
$$

where $A=\Omega \times(0, T)$ is an open set in $\boldsymbol{R}^{n+1}, z=(x, t)$, and the coefficients $a_{i j}^{\alpha \beta}(z, u)$ are supposed to be continuous in $\bar{A} \times \boldsymbol{R}^{N}$ and satisfy the ellipticity conditions:

$$
\begin{gathered}
\left|a_{i j}^{\alpha \beta}\right| \leqslant L, \\
\sum_{i, j=1}^{n} \sum_{\alpha, \beta=1}^{N} a_{i j}^{\beta \alpha} \xi_{\alpha}^{i} \xi_{\beta}^{i} \geqslant|\xi|^{2}, \quad \forall \xi \in \boldsymbol{R}^{n N} .
\end{gathered}
$$

In these hypotheses, we shall prove that every weak solution of (1.1) is regular in $A$, with the possible exception of a singular set $\Sigma$, closed in $A$. If in addition the solution $u$ belongs to the space $W_{p, 100}^{1, \frac{1}{2}}\left(A: \boldsymbol{R}^{N}\right)$ for some $p>2$, one has

$$
\mathfrak{H}_{n+2-p+\sigma}(\Sigma, \delta)=0
$$

for every $\sigma>0, \delta$ being a suitable metric in $\boldsymbol{R}^{n+1}$ and $\mathcal{H}_{\alpha}(x, \delta)$ being the $\alpha$-dimensional Hausdorff measure relative to the metric $\delta$.

(*) Entrata in Redazione il 19 settembre 1972.

(**) University of Pisa. Research supported in part by a C.N.R. fellowship at the University of Paris.

(***) University of Pisa and Stanford University. Research partially supported by NSF Grant GP 16115. 


\section{2. - Preliminaries.}

We shall consider open sets $A=\Omega \times(0, T)$ in $\boldsymbol{R}^{n+1}$, where $\Omega$ is open in $\boldsymbol{R}^{n}$ and $T>0$. A point in $\boldsymbol{R}^{n+1}$ will be denoted by $z=(x, t), x \in \boldsymbol{R}^{n}, t \in \boldsymbol{R}$. If $z_{0}=\left(x_{0}, t_{0}\right)$ is in $\boldsymbol{R}^{n+1}$, and if $R>0$, we define

$$
\begin{aligned}
& B\left(x_{0}, R\right)=\left\{x \in \boldsymbol{R}^{n}:\left|x-x_{0}\right|<R\right\}, \\
& A\left(t_{0}, R\right)=\left\{t \in \boldsymbol{R}:\left|t-t_{0}\right|<R^{2}\right\}, \\
& Q\left(z_{0}, R\right)=B\left(x_{0}, R\right) \times A\left(t_{0}, R\right) .
\end{aligned}
$$

If we introduce in $\boldsymbol{R}^{n+1}$ the metric

$$
\delta\left(z_{1}, z_{2}\right)=\max \left\{\left|x_{1}-x_{2}\right|,\left|t_{1}-t_{2}\right|^{\frac{1}{3}}\right\}
$$

then the set $Q\left(z_{0}, R\right)$ is an open ball of radius $R$, centered at $z_{0}$. We shall write $Q, Q_{R}$ instead of $Q\left(z_{0}, R\right)$ (and likewise for $B$ and $A$ ) whenever it can be done without confusion.

In addition to the usual Sobolev spaces $W_{p}^{1}(A)$, we need the following spaces:

DEFINITION 1. $-V_{p}^{1,0}(A)$ is the completion of $C^{1}(A)$ with respect to the norm:

$$
|u|_{b: A}=\left\{\int_{A}|u|^{p} d z+\sum_{i=1}^{n} \int_{A}\left|\frac{\partial u}{\partial x_{i}}\right|^{p} d z\right\}^{1 / p} \equiv\left\{\|u\|_{p, A}^{p}+\sum_{i=1}^{n}\left\|u_{x_{i}}\right\|_{p, A}^{p}\right\}^{1 / p}
$$

Definitiox 2. $-W_{p}^{1, \alpha}(A), 0<\alpha<1$, is the completion of $C^{1}(A)$ with respect to the norm:

$$
\|\| u \|_{\alpha, y ; A}=\left\{|u|_{p, A}^{p}+\int_{\Omega} d x \int_{\{0, T) \times(0, T)} \frac{|u(x, t)-u(x, s)|^{p}}{|t-s|^{1+\alpha, p}} d t d s\right\}^{1 / p} \equiv\left\{|u|_{p, A}^{p}+|u|_{(x, y) A}^{p}\right\}^{1 / p} .
$$

The following propositions are well-known:

Lemara 1. - Let $A=\Omega \times(0, T)$ be bounded and convex, and let $u \in W_{p}^{1, \frac{1}{2}}(A)$. Then:

$$
\int_{A}\left|u-u_{A}\right|^{p} d z \leqslant e_{1} \delta(A)^{p}\left\{\sum_{i=1}^{n}\left\|u_{x_{i}}\right\|_{p, A}^{p}+|u|_{i}^{p}, p ; A\right\}
$$

where

$$
u_{A}=\frac{1}{\operatorname{meas} A} \int_{A} u d z
$$

and $\delta(A)$ is the diameter of $A$ with respect to the metrio $\delta: \delta(A)=\max \{\operatorname{diam} \Omega, \sqrt{T}\}$. 
LEMna 2. - Let $A$ be as before. Then the natural imbedding of $W_{p}^{1, \frac{1}{2}}(A)$ into $L_{p}(A)$ is compact.

Finally we introduce another family of functional spaces.

Defintition 3. - Let $A=\Omega \times(0, T)$ be bounded and convex, and let $\delta$ be the metric (2.4). $\mathscr{L}^{p, \mu}(A, \delta), \mu>0$, is the space of all functions in $L_{p}(A)$ such that:

$$
[f]_{p, \mu ; A}^{p}=\sup _{\substack{z_{0} \in A \\ R>0}}\left[\operatorname{meas}\left(A \cap Q\left(z_{0}, R\right)\right)\right]^{-\mu} \int_{A \cap\left(z_{0}, R\right)}\left|f-f_{z_{0}, R}\right|^{p} d z<+\infty
$$

where

$$
f_{\tilde{z}_{0}, R}=\frac{1}{\operatorname{meas}\left(A \cap Q\left(z_{0}, R\right)\right)} \int_{A \cap Q\left(z_{0}, R\right)} f(z) d z
$$

$\mathfrak{L}^{\mathfrak{v}, \mu}(A, \delta)$ is a Banach space with norm

$$
\left\{\|f\|_{p, A}^{p}+[f]_{p, \mu: A}^{p}\right\}^{1 / p}
$$

These spaces have been introduced in [1] for the euclidean metric and in [3] for a general class of metrics including (2.4). We have the following result ([3], Theor. [3.1]).

LEMMA 3. - If $\mu>1$, then $\mathfrak{L}^{p, \mu}(A, \delta)$ is isomorphie to $O^{0, \alpha}(\Omega, \delta)$, the space of $\alpha$-hölder continuous functions with respect to the metric $\delta$, with $\alpha=((n+2) / p)(\mu-1)$.

In the following, we shall consider vector-valued functions; if $S(A)$ is a topological space of real functions in $A$, we will denote by $S\left(A ; \boldsymbol{R}^{N}\right)$ the product of $N$ copies of $S(A)$, with the natural topology. It is obvious that Lemmas 1,2 and 3 remain valid for vector-valued functions. Finally, with $S_{\text {loc }}(A)$ we denote the space of all functions $f$ in $A$ which belong to $S\left(A^{\prime}\right)$ for every $A^{\prime} \subset \subset A$.

\section{3. - Linear parabolic systems.}

In this Section we collect a number of results concerning linear parabolic systems. Results of this type are known and can be found in the literature, although sometimes in a slightly different form.

By weak solution of the parabolic system

$$
\frac{\partial u^{\alpha}}{\partial t}=\frac{\partial}{\partial x_{i}}\left(a_{i j}^{\alpha \beta}(z) \frac{\partial u^{\beta}}{\partial x_{j}}\right), \quad \alpha=1, \ldots, N,
$$

We mean a function $u \in V_{2,100}^{1,0}\left(A ; \boldsymbol{R}^{N}\right)$ such that

$$
\int_{A} u^{\alpha} \varphi_{t}^{\alpha} d z=\int_{A} a_{i j}^{\alpha \beta}(z) u_{x_{i}}^{\beta} \varphi_{x_{i}}^{\alpha} d z
$$


for every $\varphi \in C_{0}^{\infty}\left(A ; R^{N}\right)$. Here and in the following, the summation over repeated indices is understood, the latin indices $i, j, \ldots$ running from 1 to $n$, and the greek indices $\alpha, \beta, \ldots$ from 1 to $N$.

The coefficients $a_{i j}^{\alpha \beta}$ are bounded measurable functions such that

$$
\begin{array}{ll}
a_{i j}^{\alpha \beta} \xi_{\alpha}^{i} \xi_{\beta}^{j} \geqslant|\xi|^{2}=\xi_{\alpha}^{i} \xi_{\alpha}^{i}, & \forall \xi \in \boldsymbol{R}^{n N}, \forall z \in A, \\
\left|a_{i j}^{\alpha \beta}(z)\right| \leqslant L, & \forall z \in A .
\end{array}
$$

The following Lemmas are proved using methods similar to those in [7], Ch. ITI, § 4 :

LENara $4:-$ Let $u(z)$ be a weak solution of the system (3.1) in $Q_{R_{0}}$. Then $u \in$ $\in W_{2, \text { loc }}^{1, \frac{1}{2}}\left(Q_{{R_{0}}_{0}} ; \boldsymbol{R}^{N}\right)$, and for every $\varrho_{\sim}^{\dagger} R\left(0<\varrho<R<R_{0}\right)$ we have:

$$
\|u\|_{1,2: Q_{\varrho}} \leqslant c_{2}(\varrho, R) \mid u \mathbf{I}_{2, Q_{R}}
$$

Proof. - Let $\varphi^{\alpha}=\omega(t) \eta^{\alpha}(x, t), \operatorname{supp} \omega \subset A_{R}$. We get from (3.2):

$$
\int v^{\infty} \eta_{t}^{\alpha} d z=\int F_{i}^{\alpha} \eta_{x_{t}}^{\alpha} d z-\int F^{\alpha} \eta^{\alpha} d z
$$

where

$$
v^{\alpha}=\omega u^{\alpha} ; \quad F_{i}^{\alpha}=a_{i j}^{\alpha \beta} v_{x j}^{\beta} ; \quad F^{\alpha}=u^{\alpha} \omega
$$

Now let $\beta_{\varepsilon}(t)$ be a mollifier, $\beta_{\varepsilon}(t)=\beta_{\varepsilon}(-t)$; if we put $\eta(x, t)=\beta_{\varepsilon} * \theta=\theta_{\varepsilon}(x, t)$, we obtain

$$
\int v_{\varepsilon}^{\alpha} \theta_{i}^{\alpha} d z=\int F_{i, \varepsilon}^{\alpha} \theta_{x_{i}}^{\alpha} d z-\int F_{\varepsilon}^{\alpha} \theta^{\alpha} d z
$$

In particular, if $\theta^{\alpha}(x, t)=\gamma(t) \psi^{\alpha}(x)$, with $\gamma \in \mathcal{D}\left(\Lambda_{R}\right)$ and $\psi^{\alpha} \in \stackrel{\circ}{W}_{2}^{1}\left(B_{R}\right)$, we get the equation

$$
\int\left\langle v_{\varepsilon}, \psi\right\rangle \gamma^{\prime} d t=\int\left\{\left\langle F_{i, e}, w_{x_{i}}\right\rangle-\left\langle F_{s}, \psi\right\rangle\right\} \gamma d t
$$

where

$$
\langle f, g\rangle=\int f^{\alpha} g^{\alpha} d x
$$

and hence, in the sense of distributions,

$$
\frac{d}{d t}\left\langle\boldsymbol{v}_{\varepsilon}, \psi\right\rangle=-\left\langle F_{i, \varepsilon}, \psi_{x_{i}}\right\rangle+\left\langle F_{\varepsilon}, \psi\right\rangle
$$

Now let $\hat{f}(\tau)$ denote the Fourier transform of $f(t)$. We have

$$
i \tau\left\langle\hat{v}_{\varepsilon}, \psi\right\rangle=\left\langle\hat{F}_{i, \varepsilon}, \psi_{x_{i}}\right\rangle-\left\langle\hat{F}_{\varepsilon}, \psi\right\rangle
$$


for every $\tau \in \boldsymbol{R}$ and every $\psi \in \stackrel{\circ}{W}_{2}^{1}\left(B_{R}\right)$. In particular, we can choose

$$
\psi=-i \operatorname{sign} \tau \widehat{v}_{\boldsymbol{\varepsilon}} \sigma(x)^{2}
$$

with $\sigma \in \mathscr{D}\left(B_{R}\right)$; integrating with respect to $\tau$, we easily get:

$$
\int|\tau|\left|\sigma \hat{v}_{\varepsilon}\right|^{2} d x d \tau \leqslant\left\|\sigma F_{i, \varepsilon}\right\|_{2}\left\{\left\|\sigma v_{\varepsilon, x}\right\|_{2}+\left\|\sigma_{x} v_{\varepsilon}\right\|_{2}\right\}+\left\|\sigma F_{\varepsilon}\right\|_{2}\left\|\sigma v_{\varepsilon}\right\|_{2}
$$

Finally, if we let $\varepsilon \rightarrow 0$ (remember that for every function $g(x, t)$ with compact support, we have

$$
\left.\int d x \int \frac{|g(x, t)-g(x, s)|^{2}}{|t-s|^{2}} d t d s=2 \int_{-\infty}^{\infty} \frac{\sin ^{2} t}{t^{2}} d t \int|\tau||\hat{g}|^{2} d \tau d x\right),
$$

and choose $\omega$ and $\sigma$ in such a way that $\omega \sigma \equiv 1$ on $Q_{\varrho}$, we easily get the conclusion of the lemma.

q.e.d.

Lemrua 5. - With the hypotheses of Lemma 4, we have

$$
\mid u \mathbf{|}_{2, Q_{\varrho}} \leqslant c_{3}(\varrho, R)\|u\|_{2, Q_{R}}
$$

for every $R, \varrho\left(0<\varrho<R<R_{0}\right)$.

Proof. - If $\varphi=\beta_{\varepsilon} * \theta$, we have from (3.2)

$$
\int_{Q_{R}} u_{\varepsilon}^{\alpha} \theta_{l}^{\alpha} d z=\int_{Q_{R}}\left(a_{i j}^{\alpha \beta} u_{x j}^{\beta}\right)_{e} \theta_{x_{l}}^{\alpha} d z
$$

In particular, if $\theta=\sigma^{2}(x) \omega^{2}(t) u_{s}$, with $\sigma(x) \in \mathfrak{D}\left(B_{R}\right)$ and $\omega(t) \in \mathfrak{D}\left(\Lambda_{R}\right)$, we get

$$
0=\frac{1}{2} \int_{B_{R}} \sigma^{2} d x \int_{A_{R}} \frac{d}{d t}\left|\omega u_{\varepsilon}\right|^{2} d t=\int_{Q_{R}}\left(a_{i j}^{\alpha \beta} u_{x_{j}}^{\beta}\right)_{\varepsilon} \omega^{2}\left(\sigma^{2} u_{\varepsilon}^{\alpha}\right)_{x_{i}} d z-\int_{Q_{R}}\left|u_{\varepsilon}\right|^{2} \omega \omega_{t} \sigma^{2} d z
$$

whence, if $\varepsilon \rightarrow 0$,

$$
\int a_{i j}^{\alpha \beta} u_{x_{j}}^{\beta} u_{x_{t}}^{\alpha} \omega^{2} \sigma^{2} d z=\int|u|^{2} \omega \omega_{t} \sigma^{2} d z-2 \int a_{i j}^{\alpha \beta} u_{x_{j}}^{\beta} \omega^{2} \sigma^{\alpha} \sigma_{x_{t}} d z .
$$

With the usual choice of $\sigma$ and $\omega$, it is not difficult to get (3.13) from (3.15). q.e.d. An immediate consequence of the two previous lemmas is

Corollary 1. - Let $u$ be as before. Then for every $\varrho, R\left(0<\varrho<R<R_{0}\right)$, we have

$$
\|u\|_{1,2 ; Q_{Q}} \leqslant e_{4}(\varrho, R)\|u\|_{2, Q_{n}}
$$

17 - Annali di Matematica 
Let us now recall a result concerning systems with constant coefficients. The proof can be found in [2].

LEMMA 6. - Let $u(t)$ be a weak solution of (3.1) in $Q$, and let the coefficients $a_{i j}^{\alpha \beta}$ be constant in $Q_{1}$. Suppose further that $u \in L_{2}\left(Q_{1}, \boldsymbol{R}^{N}\right)$. Then for every $\varrho<1$, we have

$$
\frac{1}{\operatorname{meas} Q_{Q}} \int_{Q_{Q}}\left|u-u_{Q_{e}}\right|^{2} d z \leqslant c_{5} \varrho^{2} \frac{1}{\operatorname{meas} Q_{1}} \int_{Q_{1}}\left|u-u_{Q_{1}}\right|^{2} d z \text {. }
$$

We conclude this Section with a simple result concerning the convergence of solutions of parabolic systems.

LEMMA 7. - Let $a_{i j}^{\alpha \beta(v)}(z)(v=1,2, \ldots)$ be a sequence of bounded measurable functions in $Q_{1}$, verifying (3.2) with $L$ independent of $v$, and such that

$$
\lim _{\nu \rightarrow \infty} a_{i j}^{\alpha \beta(\nu)}(z)=a_{i j}^{\alpha \beta}(z) \quad \text { a.e. in } Q_{1} .
$$

Let $u^{(\nu)}$ be a sequence of funotion in $L_{2}\left(Q_{1} ; \boldsymbol{R}^{N}\right)$, weak solutions in $Q_{1}$ of the system (3.1) with coefficients $a_{i j}^{\alpha \beta(v)}$ :

$$
\int_{Q_{2}} u^{(\nu) \alpha} \varphi_{i}^{\alpha} d z=\int_{Q_{1}} a_{i j}^{\alpha \beta(v)}(z) u_{x_{j}}^{(v) \beta} \varphi_{x_{t}}^{\alpha} d x, \quad \forall \varphi \in C_{0}^{\infty}\left(Q_{1}, \boldsymbol{R}^{N}\right)
$$

Suppose further that

$$
\lim _{\nu \rightarrow \infty} u^{(\nu)}=u \quad \text { weakly in } L^{2}\left(Q_{1} ; \boldsymbol{R}^{N}\right) .
$$

Then $u \in V_{2,100}^{\mathbf{1}, 0}\left(Q_{1}, \boldsymbol{R}^{N}\right)$, and for every $R<1$ we have

$$
\begin{array}{ll}
\lim _{\nu \rightarrow \infty} u^{(v)}=u & \text { strongly in } L_{2}\left(Q_{R}, \boldsymbol{R}^{N}\right), \\
\lim _{\nu \rightarrow \infty} u_{x_{t}}^{(p)}=u_{x_{t}} & \text { weakly in } L_{2}\left(Q_{R}, \boldsymbol{R}^{N}\right)
\end{array}
$$

$(i=1, \ldots, n)$. In addition, $u(z)$ is a weak solution of the system

$$
\int_{Q_{1}} u^{\alpha} \varphi_{t}^{\alpha} d z=\int_{Q_{1}} a_{i j}^{\alpha \beta}(z) u_{x_{j}}^{\beta} \varphi_{x_{i}}^{\alpha} d z, \quad \forall \varphi \in C_{0}^{\infty}\left(Q_{1}, \boldsymbol{R}^{N}\right)
$$

Proof. - It is easily seen that (3.20) and Lemma 5 imply (3.22), while (3.21) follows from Corollary 1, Lemma 2, and (3.20). Finally, letting $y \rightarrow \infty$ in (3.19), one gets $(3.23)$ at once. 


\section{4. - The regularity of solutions of parabolic systems.}

We shall consider solutions of nonlinear parabolic systems of partial differential equations:

$$
\int_{A} u^{\alpha} \varphi_{t}^{\alpha} d z=\int_{A}^{\alpha \beta} a_{i j}^{\alpha \beta}(z, u) u_{x_{j}}^{\beta} \varphi_{x_{i}}^{\alpha} d x, \quad \forall \varphi \in C_{0}^{\infty}\left(A, \boldsymbol{R}^{N}\right)
$$

where, as usual, $A=\Omega \times(0, T)$ is an open set in $\mathbf{R}^{n+1}, z=(x, t), x \in \Omega, 0<t<T$, and we sum over repeated indices, the latin indices running from 1 to $n$, and the greek indices from 1 to $N$.

The coefficients $a_{i j}^{\alpha \beta}(z, u)$ are supported to be continuous functions in $\bar{A} \times \boldsymbol{R}^{N}$, and to satisfy the ellipticity conditions:

i) $\left|a_{i j}^{\alpha \beta}(z, u)\right| \leqslant L, \forall z \in \bar{A}, u \in \boldsymbol{R}^{N}$,

ii) $a_{i j}^{\alpha \beta}(z, u) \xi_{\alpha}^{i} \xi_{\beta}^{j}=|\xi|^{2}, \forall \xi \in \boldsymbol{R}^{n N}, z \in \bar{A}, u \in \boldsymbol{R}^{N}$.

We have the following

Lencra 8. - For every $\tau, 0<\tau<1$, and for every $M>0$, there exist two constants $\varepsilon_{0}$ and $R_{0}$ such that if $u(z)$ is a weak solution of (4.1), and if for some $z_{0} \in A$ and some $R<R_{0} \cap \delta\left(z_{0}, \partial A\right)(a \cap b=\min \{a, b\})$, we have

$$
U\left(z_{0}, R\right) \equiv \frac{1}{\operatorname{meas} Q_{R}} \int_{Q\left(z_{0}, R\right)}\left|u-u_{Q\left(z_{0}, R\right)}\right|^{2} d z<\varepsilon_{0}^{z}
$$

and

$$
\left|u_{Q\left(z_{0}, R\right)}\right| \leqslant M
$$

then:

$$
U\left(z_{0}, \tau R\right) \leqslant 2 e_{5} \tau^{2} U\left(z_{0}, R\right)
$$

where $c_{5}$ is the constant appearing in Lemma 5.

Proof. - Suppose the lemma is false. Then for some $\tau$ and $M$, there exist sequences $z_{v} \in A, \varepsilon_{v} \rightarrow 0, R_{v} \rightarrow 0$, and a sequence $u^{(v)}$ of weak solutions of (4.1) such that

$$
\begin{aligned}
& \left|u_{Q\left(z_{y}, R_{y}\right)}^{(v)}\right| \leqslant M, \\
& U^{(\nu)}\left(z_{\nu}, R_{\nu}\right)=\varepsilon_{\nu}^{2},
\end{aligned}
$$

and

$$
U\left(z_{v}, \tau R_{p}\right)>2 c_{5} \tau^{2} \varepsilon_{2}^{p}
$$


If we define

$$
v^{(v)}(z)=v^{(v)}(x, t)=\varepsilon_{v}^{-1}\left(u^{(v)}\left(x_{y}+R_{p} x, t_{v}+R_{v}^{2} t\right)-u_{Q\left(z_{v}, R_{p}\right)}^{(v)}\right\},
$$

we have $(Q=Q(0,1))$ :

$$
\int_{Q} v^{(\nu) \alpha} \varphi_{t}^{\alpha} d z=\int_{Q} a_{i j}^{\alpha \beta}\left(x_{v}+R_{v} x, t_{v}+R_{v}^{2} t ; \varepsilon_{v} v^{(v)}+u_{Q\left\{x_{v}, R_{v}\right)}^{(\nu)}\right) v_{x_{j}}^{(p) \beta} \varphi_{x_{t}}^{\alpha} d z, \quad \forall \varphi \in C_{0}^{\infty}\left(Q ; \boldsymbol{R}^{N}\right)
$$

and, from $(4.6),(4.7)$ :

$$
\begin{aligned}
& V^{(\nu)}(0,1)=\frac{1}{\operatorname{meas} Q} \int_{Q}\left|v^{(v)}\right|^{2} d z=1, \\
& V^{(v)}(0, \tau)>2 c_{5} \tau^{2} .
\end{aligned}
$$

From (4.10), passing in case to a subsequence, we get

$$
\begin{array}{ll}
v^{(p)} \rightarrow v & \text { weakly in } L_{2}\left(Q ; \boldsymbol{R}^{N}\right), \\
\varepsilon_{v} v^{(v)} \rightarrow 0 & \text { a.e. in } Q, \\
z_{v} \rightarrow \bar{z} ; & u_{Q\left(x_{v}, R_{v}\right)}^{(v)} \rightarrow \lambda,
\end{array}
$$

whenee

$$
a_{i j}^{\alpha \beta}\left(x_{\nu}+R_{v} x, t_{v}+R_{v}^{2} t ; \varepsilon_{\nu} v^{(\nu)}+u_{Q\left(x_{y}, R_{\nu}\right)}^{(v)}\right) \rightarrow a_{i j}^{\alpha \beta}(\bar{z}, \lambda) \quad \text { a.e. in } Q .
$$

It follows from Lemma 6 that the function $v$ is a weak solution in $Q$ of the system:

$$
\int_{Q} v^{\alpha} \varphi_{t}^{\alpha} d z=\int_{Q} a_{i j}^{\alpha \beta}(\bar{z}, \lambda) v_{x_{j}}^{\beta} \varphi_{x_{i}}^{\alpha} d z, \quad \forall \varphi \in C_{0}^{\infty}\left(Q ; \boldsymbol{R}^{N}\right)
$$

whence, from Lemma 5,

$$
\nabla(0, \tau) \leqslant c_{5} \tau^{2} V(0,1)
$$

On the other hand, passing to the limit in (4.10), (4.11), we get

$$
\begin{aligned}
& V(0,1) \leqslant \liminf _{\nu \rightarrow \infty} V^{(\nu)}(0,1)=1, \\
& V(0, \tau)=\lim _{\nu \rightarrow \infty} V^{(\nu)}(0, \tau) \geqslant 2 c_{5} \tau^{2},
\end{aligned}
$$

and these inequalities contradict (4.16).

q.e.d.

The constants $\varepsilon_{0}$ and $R_{0}$ in the preceding lemma depend on $\tau, M, n, N$, and on the coefficients $a_{i j}^{\alpha \beta}$. In the following, we shall emphasize the dependence on $M$ by 
writing $\varepsilon_{0}(M), R_{0}(M)$. The following lemma is essentially an iteration of the preceding.

LeMnad 9. - Let $\tau<\left(2 c_{5}\right)^{-\frac{1}{2}}$, and let u be a weak solution of (4.1) such that for some $z_{0} \in A$ and for some $R<R_{0}(2 M) \cap \delta\left(z_{0}, \partial A\right)$, we have

$$
\begin{aligned}
& \left|u_{Q\left(z_{6}, R\right)}\right| \leqslant M, \\
& U\left(z_{0}, R\right)<\eta_{0}^{2}(M),
\end{aligned}
$$

where

$$
\eta_{0}(M)=\min \left\{\varepsilon_{0}(2 M), M \tau^{1+\infty / 2}\left(1-\tau \sqrt{2 c_{5}}\right)\right\}
$$

Then for every integer $k \geq 0$, we have

$$
U\left(\tilde{z}_{0}, \tau^{k} R\right) \leqslant\left(2 c_{5} \tau^{2}\right)^{k} U\left(z_{0}, R\right) .
$$

Proof. - We have, for every $\varrho>0$,

$$
\left|u_{Q\left(z_{0}, \tau \ell\right.}-u_{Q\left(z_{0}, \varrho\right)}\right| \leqslant \tau^{-1-n / 2} U\left(z_{0}, \varrho\right)^{\frac{1}{2}},
$$

whence

$$
\left|u_{Q\left(z_{B}, \tau^{k} R\right)}\right| \leqslant\left|u_{Q\left(z_{\theta}, R\right)}\right|+\tau^{-1-n / 2} \sum_{j=0}^{k-1} U\left(z_{0}, \tau^{j} R\right)^{\frac{1}{z}}
$$

For $k=0$, the inequality (4.22) is trivial. Suppose now that $(4.22)_{k}$ is true for every $h \leqslant k$. From (4.24) and (4.21), we easily get

$$
\left|u_{Q\left(z_{0}, z^{k} R\right)}\right| \leqslant 2 M,
$$

while from $(4.22)_{k}$, recalling that $2 c_{5} \tau^{2}<1$, we obtain at once

$$
U\left(z_{0}, \tau^{k} R\right)<\varepsilon_{0}^{2}(2 M)
$$

From (4.25), (4.26), and the preceding Lemma, we get $(4.22)_{k+1}$.

q.e.d.

We can now prove the first part of the regularity theorem.

THEOREM 1. - For every weak solution $u(z)$ of (4.1), there exists an open set $A_{0} \subset A$ such that $u(z)$ is (locally) $\alpha$-hölder continuous (with respect to the metrio $\delta$ ) in $A_{0}$, for every $\alpha<1$.

Proof. - Let $z_{0} \in A$ be such that

$$
\begin{gathered}
\sup _{0<R<\delta\left(z_{0}, \partial A\right)}\left|u_{Q\left(z_{0}, R_{i}\right)}\right| \leqslant M / 2<+\infty, \\
\liminf _{R \rightarrow 0^{+}} U\left(z_{0}, R\right)=0 .
\end{gathered}
$$


Let $\alpha=1-\sigma, 0<\sigma<1$, and let $\tau=\left(2 e_{5}\right)^{-\frac{1}{6} \sigma}$. There exists an $R<R_{0}(2 M) \cap$ $\cap \delta\left(z_{0}, \delta A\right)$ such that

$$
U\left(z_{0}, R\right)<\eta_{0}^{2}(M)
$$

Since $U(z, r)$ and $u_{Q(z, r)}$ are continuous functions of $z$ and $r(r>0)$, there exists an $s$, $0<s<R / 2$, such that for every $z \in Q\left(\tilde{z}_{0}, s\right)$, we have

$$
\begin{aligned}
& \left|u_{Q\left(z, R_{z}\right.}\right| \leqslant M, \\
& U\left(z, R_{z}\right)<\eta_{0}^{2}(M),
\end{aligned}
$$

with $R_{z}=R-\delta\left(z, z_{0}\right)>R / 2$.

From (4.29), (4.30), and Lemma 8, we get for every $k$ :

$$
U\left(z, \tau^{k} R_{z}\right) \leqslant\left(2 c_{5} \tau^{2}\right)^{k} U\left(z, R_{z}\right) \leqslant \tau^{2 k(1-\sigma)} \eta_{0}^{2}
$$

For every $\varrho, 0<\varrho<R / 2<R_{z}$, let $h$ be the integer such that

$$
\tau^{n+1} R_{z}<\varrho \leqslant \tau^{h} R_{z}
$$

We have

$$
U(z, \varrho) \leqslant\left(\frac{\varrho}{\tau^{h+1} R_{z}}\right)^{n+2} U(z, \varrho) \leqslant \tau^{-n-2} U\left(z, \tau^{h} R_{z}\right) \leqslant \tau^{-n-4+2 \sigma}\left(\frac{\varrho}{R_{x}}\right)^{2(1-\sigma)} \eta_{0}^{2},
$$

whence

$$
U(z, \varrho) \leqslant\left(\frac{2}{R}\right)^{2-2 \sigma} \eta_{0}^{2}\left(2 c_{5}\right)^{(1 n+a) / 2 \sigma)-1} \varrho^{2(1-\alpha)}
$$

for every $z \in Q\left(z_{0}, s\right)$ and every $\varrho<R / 2$.

It is easily seen that (4.33) implies that $u(x)$ belongs to $\mathfrak{L}^{2, \mu}\left(Q\left(z_{0}, s\right), \delta\right)$ with $\mu=1+(2 /(n+2))(1-\sigma)$, so that the theorem follows at once from Lemma 3 . q.e.d.

REMARK 1. - The set $\Sigma=A-A_{0}$, which is closed in $A$, will be called the singular set of $u(z)$. A point $z_{0}$ is not in $\Sigma$ if and only if (4.27) and (4.28) are satisfied.

With minor charges in the argument, one can show as in [6] that if the coeffcients $a_{i j}^{\alpha \beta}$ are uniformly continuous in $\bar{A} \times \boldsymbol{R}^{N}$, then (4.28) alone is sufficient for the regularity of $z_{0}$.

\section{5. - The singular set.}

In this Section, we shall obtain an upper bound for the Hausdorff dimension of the singular set $\Sigma$. 
LEMMA 10. - Let $u(z)$ be a function in $L_{2}(A)$, and let $z_{0} \in A$ be suoh that

$$
U\left(z_{0}, R\right) \leqslant c_{6} R^{2 \varepsilon}
$$

for some $\varepsilon>0$ and some $e_{6}$ depending on $u$ and $z_{0}$. Then there exists, and is finite, the limit

$$
\lim _{Q \rightarrow 0^{+}} u_{Q\left(z_{0}, Q\right)} .
$$

Proor. - Let $f(r)=u_{Q\left(z_{0}, r\right)}$. We have, for $z \in A$ and $0<\sigma<\varrho<\delta\left(z_{0}, \partial A\right)$,

$$
|f(\varrho)-f(\sigma)|^{2} \leqslant 2\left\{|u(z)-f(\varrho)|^{2}+\left.|u(z)-f(\sigma)|\right|^{2}\right\} .
$$

Integrating over $Q\left(z_{0}, \sigma\right)$,

$$
\sigma^{n+2}|f(\varrho)-f(\sigma)|^{2} \leqslant c_{7}\left\{\varrho^{n+2} U\left(z_{0}, \varrho\right)+\sigma^{n+2} U\left(z_{0}, \sigma\right)\right\},
$$

whence, using (5.1),

$$
|f(\varrho)-f(\sigma)| \leqslant c_{8} \sigma^{-1-n / 2}\left\{\varrho^{n+2+2 e}+\sigma^{n+2+2 \varepsilon}\right\}^{\frac{1}{2}} .
$$

In particular, we have

$$
\left|f\left(2^{-i} \varrho\right)-f\left(2^{-i-1} \varrho\right)\right| \leqslant o_{9} 2^{-i \varepsilon} \varrho^{\varepsilon},
$$

and, if $h<k$,

$$
\left|f\left(2^{-h} \varrho\right)-f\left(2^{-k} \varrho\right)\right| \leqslant c_{9} \varrho^{\varepsilon} \sum_{j=h}^{k-1} 2^{-j \varepsilon}
$$

so that the sequence $f\left(2^{-h} \varrho\right)$ converges, as $h \rightarrow \infty$, to some real number $\lambda$. It is easily seen that $\lambda$ does not depend on $\varrho$; in fact, from (5.2) we get

$$
\left|f\left(2^{-i} \varrho\right)-f\left(2^{-i} r\right)\right| \leqslant c_{8}\left\{\frac{\varrho^{n+2+2 \varepsilon}+r^{n+2+2 \varepsilon}}{\max [\varrho, r]^{n+2}}\right\}^{\frac{1}{2}} 2^{-i \varepsilon},
$$

and the right-hand side of (5.4) tends to zero as $i \rightarrow \infty$. Finally, choosing $h=0$ in (5.3) and letting $k \rightarrow \infty$, we get

$$
|f(\varrho)-\lambda| \leqslant c_{10} \varrho^{2},
$$

which proves the lemma.

q.e.d,

REMARK 2. - The preceding lemma and Remark 1 show that a point $z_{0}$ is regular if there exist constants $\varepsilon>0$ and $c_{5}$ such that

$$
U\left(z_{0}, R\right) \leqslant e_{6} R^{28}
$$


for every $R<\delta\left(z_{0}, \partial A\right)$; or, what is the same, if there exists a $\sigma>0$ such that

$$
\lim _{R \rightarrow 0^{+}} R^{-\sigma} U\left(z_{0}, R\right)=0
$$

Let us observe that if the coefficients are uniformly continuous, then it is sufficient to require (5.5) with $\sigma=0$. This will give a slight difference between the two cases.

Let us now recall the Hausdorff measure.

Definition 4. - Let $\delta$ be the metrie (2.4), and let $\alpha$ be a non-negative real number, The $\alpha$-dimensional Hausdorff measure of a set $X \subset \boldsymbol{R}^{n+1}$, with respect to the metric $\delta$. is given by

$$
\mathscr{H}_{\alpha}(X ; \delta)=\lim _{\varepsilon \rightarrow 0^{+}} \inf \left\{\sum_{i} \delta\left(x_{i}\right)^{\alpha} ; \bigcup_{i} X_{i} \supset X ; \delta\left(X_{i}\right)<\varepsilon\right\}
$$

The $\delta$-Hausdorff dimension of a set $X$ is the infimum of the numbers $\alpha$ such that $\mathfrak{K}_{\alpha}(X ; \delta)=0$.

The next lemma is a simple modification of Theorem 1 of [5].

LEMMA 11. - Let $f \in L_{1, \mathrm{loo}}(A)$, and, for $0<\alpha<n+2$, let

Then we have

$$
F_{\alpha}=\left\{z_{0} \in A: \limsup _{\varrho \rightarrow 0^{+}} \varrho^{-\alpha} \int_{Q\left(z_{0}, \varrho\right)}|f| d z>0\right\} .
$$

$$
\mathscr{H}_{\alpha}\left(F_{\alpha}^{\prime}, \delta\right)=0
$$

The following result concludes the proof of the regularity theorem.

THEOREM 2. - Let $u \in W_{p, 100}^{1, \frac{\pi}{3}}\left(A ; \boldsymbol{R}^{n}\right), p \geqslant 2$, be a weak solution of (4.1). Then for every $\sigma>0$, we have

$$
\mathscr{H}_{n+2-p+\sigma}(\Sigma, \delta)=0
$$

If the coefficients are uniformly continuous, we can take $\sigma=0$ in (5.6).

ReMarK 3. - From Lemma 4, it follows at once that for every weak solution $u(z)$ of (4.1), we have $\mathscr{H}_{n+\sigma}(\Sigma)=0, \forall \sigma>0$.

Proof. of Theorem 2. - According to Remark 2, it is sufficient to prove that $\mathfrak{J}_{n+2-p+\sigma}\left(E_{\sigma}, \delta\right)=0$, where

We have

$$
E_{\sigma}=\left\{z_{0} \in A: \limsup _{R \rightarrow 0^{+}} R^{-2 \sigma / p} U\left(z_{0}, R\right)>0\right\} .
$$

$$
R^{-2 \sigma / p} U\left(z_{0}, R\right) \leqslant 0_{\perp 1}\left\{R^{-n-2-\sigma} \int_{Q\left(z_{0}, R\right)}\left|u-u_{Q\left(z_{0}, R\right)}\right|^{p} d z\right\}^{2 / p},
$$


and, by Lemma 1,

$$
\begin{aligned}
R^{-2 \sigma / p} U\left(z_{0}, R\right) \leqslant c_{12}\left\{\left[R^{p-2-n-\sigma} \int_{Q\left(z_{0}, R\right)} \sum_{i=1}^{n}\left|u_{x_{i}}\right|^{p} d z\right]^{2 / p}+\right. \\
\left.+\left[R^{p-n-2-\sigma} \int_{B\left(x_{0}, R\right)} d x \int_{A\left(t_{0}, R\right) \times\left(A\left(t_{0}, R\right)\right.} \frac{|u(x, t)-u(x, s)|^{p}}{|t-s|^{1+n / 2}} d t d s\right]^{2 / p}\right\} .
\end{aligned}
$$

The functions $\left|u_{\alpha_{i}}\right|^{p}$ belong to $L_{1, \text { loo }}(A)$, whence, by Lemma 11, the first integral at the right-hand side tends to zero $\mathfrak{H}_{n+2-p+\sigma}$ almost everywhere.

For the second integral, we observe that the function

$$
f(x, s, t)=\frac{|u(x, t)-u(x, s)|^{p}}{|t-s|^{1+p / 2}}
$$

belongs to $L_{1, \text { loc }}(\tilde{A}), \tilde{A}=\Omega \times(0, T) \times(0, T)$. If we denote by $\xi=(x, t, s)$ a point in $\tilde{A}$, by $\tilde{\delta}$ the metrie

$$
\tilde{\delta}\left(\xi_{1}, \xi_{2}\right)=\max \left\{\left|x_{1}-x_{2}\right|,\left|t_{1}-t_{2}\right|^{\frac{1}{2}},\left|s_{1}-s_{2}\right|^{\frac{1}{2}}\right\},
$$

and by $\widetilde{Q}$ the ball in the metric $\tilde{\delta}$, we get easily that

$$
R_{\tilde{Q}(\xi), R\rangle}^{p-n-2-\sigma} \int_{\xi} f(\xi) d \xi
$$

tends to zero $\widetilde{\mathfrak{H}}_{n+2-p+\sigma}$ almost everywhere. From that remark, it follows at once that $\mathscr{H}_{n+2-D+\sigma}\left(E_{\sigma}, \delta\right)=0$.

q.e.d.

In the case of elliptic systems in $\boldsymbol{R}^{n}$, one was able to prove (see [6] and [5]) that

$$
H_{n-p+\sigma}(\Sigma)=0, \quad \forall \sigma>0,
$$

for continuous coefficients, and

$$
H_{n-s}(\Sigma)=0
$$

with the assumption of uniform continuity, $H_{\alpha}$ being the $\alpha$-dimensional Hausdorff measure with respect to the usual metric in $\boldsymbol{R}^{n}$. Comparing (5.8) and (5.9) with the conclusion of Theorem 2, it seems at first that the introduction of the time variable gives a jump of two in the dimension of the singular set. The following argument shows that this is not the case. Let us first observe that the restriction of the metric $\delta$ to the $t$-axis gives the metric $d\left(t_{1}, t_{2}\right)=\left|t_{1}-t_{2}\right|^{\frac{1}{2}}$ so that the Hausdorff measure $\mathfrak{H}_{\alpha}(X, d)$ verifies

$$
\mathcal{H}_{\alpha}(X, d)=H_{\alpha / 2}(X), \quad \forall X \subset \boldsymbol{R}_{i},
$$

$H_{\beta}$ being the usual Hausdorff measure in $\boldsymbol{R}^{1}$. 
On the other hand, $\delta$ coincides with the usual metric when restricted to $\boldsymbol{R}^{n}$, so that the Hausdorff measure is also the same:

$$
\mathscr{H}_{\alpha}(X, \delta)=X_{\alpha}(X), \quad \forall X \subset \boldsymbol{R}_{x}^{n}
$$

Finally, if $\pi_{1}$ and $\pi_{2}$ are the projection operators on $\boldsymbol{R}$ and $\boldsymbol{R}^{n}$, respectively, we have the following inequality ([4], Th. 2.10.25):

$$
\int_{\mathbf{R}}^{*} H_{\alpha}\left\{\pi_{2}\left(\Sigma \cap \pi_{1}^{-1}(t)\right)\right\} d H_{\beta / 2}(t) \leqslant \mathscr{H}_{\alpha+\beta}(\Sigma, \delta)
$$

for every non-negative $\alpha, \beta$.

In particular, if we choose $\beta=2$ and $\alpha=n-p+\sigma$, we conclude from (5.10) and (5.6) that

$$
H_{n+\sigma-p}\left(\Sigma \cap \pi_{1}^{-1}(t)\right)=0 \quad \text { for almost every } t
$$

A similar result, with $\sigma=0$, holds for uniformly continuous coefficients.

\section{REFERENCES}

[1] S. Campanato, Preprietà di hölderianità di alcune classi di funzioni, Ann. Scuola Norm. Sup. Pisa, 17 (1963), pp. 175-188.

[2] S. Campanato, Equazioni paraboliche del secondo ordine e spazi $\mathcal{L}^{2, \theta}(\Omega, \delta)$, Ann. di Mat. pura e appl., 73 (1966), pp. 55-102.

[3] G. DA Prato. Spazi $\mathcal{L}^{(p, \theta)}(\Omega, \delta)$ e loro proprietà, Ann. di Mat. pura e appl., 69 (1965), pp. 383-392.

[4] H. Federen, Geometric measure theory, Springer-Verlag, 1969.

[5] E. GrUstr, Precisazione delle funzioni in $H^{1, p}$ e singolarità nelle soluzioni deboli di sistemi ellittici non lineari, Boll. U.M.I., 1 (1969), pp. 71-76.

[6] E. Grusti - M. Mrranda, Sulla regolarità nelle soluzioni deboli di una classe di sistemi ellittici quasi-lineari, Arch. Rat. Mech. Anal, 31 (1968), pp. 173-184.

[7] O. A. LADXŽTNSKAJA - V. A. SOLONNIKov - N. N. URAL'CEVA, Linear and quasilinear operators of parabolic type, Am. Math. Soc. (1968). 